\title{
Fragrance chemistry and pollinator affinities in Nyctaginaceae
}

\author{
Rachel A. Levin ${ }^{\mathrm{a}, *, 1}$, Robert A. Raguso ${ }^{\mathrm{b}}$, Lucinda A. McDade \\ ${ }^{a}$ Department of Ecology and Evolutionary Biology, University of Arizona, Tucson, AZ 85721, USA \\ ${ }^{\mathrm{b}}$ Department of Biology, Coker Life Sciences Building, University of South Carolina, Columbia, SC 29208, USA \\ cAcademy of Natural Sciences, 1900 Ben Franklin Parkway, Philadelphia, PA 19103, USA
}

Received 5 December 2000; received in revised form 11 May 2001

\begin{abstract}
We present results of dynamic head-space collections and GC-MS analyses of floral and vegetative fragrances for 20 species in three genera of Nyctaginaceae: Acleisanthes, Mirabilis and Selinocarpus. Most of the species included in this study are either hawkmoth or noctuid moth-pollinated. A wide variety of compounds were observed, including mono- and sesquiterpenoids, aromatics (both benzenoids and phenylpropanoids), aliphatic compounds, lactones, and nitrogen-bearing compounds. Intraspecific variation in fragrance profiles was significantly lower than interspecific variation. Each species had a unique blend of volatiles, and the fragrance of many species contained species-specific compounds. The fragrance profiles presented here are generally consistent with previous studies of fragrance in a variety of moth-pollinated angiosperms. (C) 2001 Elsevier Science Ltd. All rights reserved.
\end{abstract}

Keywords: Acleisanthes; Mirabilis; Selinocarpus; Nyctaginaceae; Four o'clock; Floral fragrance; Pollination; Hawkmoths

\section{Introduction}

In many pollination interactions, floral fragrance appears to act in concert with visual cues to attract floral visitors (see review by Dobson, 1994). Consequently, a number of studies have examined the relationship of fragrance composition to type of floral visitor (Knudsen and Tollsten, 1993, 1995; Borg-Karlson et al., 1994; Kaiser and Tollsten, 1995; Bestmann et al., 1997; Dobson et al., 1997; Miyake et al., 1998; Grison et al., 1999). The fragrances of hawkmoth-pollinated flowers in a number of plant families have been of particular interest (Knudsen and Tollsten, 1993; Kaiser and Tollsten, 1995; Raguso and Pichersky, 1995; Barthlott et al., 1997; Miyake et al., 1998). Such flowers often have similar morphologies, with long, tubular perianths that are usually white, occasionally yellow or pink (Grant, 1983; Haber and Frankie, 1989). Because these flowers generally emit fragrance at night, when most hawkmoths

\footnotetext{
* Corresponding author. Tel.: +1-301-238-3444, x114; fax: +1301-238-3059.

E-mail address: rlevin@lms.si.edu (R.A. Levin).

1 Current address: Laboratory of Molecular Systematics, Smithsonian Museum Support Center, 4210 Silver Hill Road, Suitland, MD 20746, USA.
}

are active, it has been suggested that convergent evolution for hawkmoth attraction in various angiosperm families may involve scent chemistry as well as floral morphology (Nilsson et al., 1985; Knudsen and Tollsten, 1993; Miyake et al., 1998). To date there has been some support for this idea (Kaiser, 1993; Knudsen and Tollsten, 1993; Miyake et al., 1998); oxygenated terpenoids and nitrogen-bearing compounds are frequently present in the fragrance of hawkmoth-pollinated flowers. However, the high degree of among species variation in fragrance chemistry identified in these studies suggests that convergence is but one of several factors affecting scent chemistry.

Nyctaginaceae are a small family of trees, shrubs, and herbs, distributed mainly in tropical and subtropical regions of the New World. This family is commonly called the Four o'clock family, as most species have flowers that open in the late afternoon to early evening. Further, the flowers of many species are pollinated by hawkmoths (Grant, 1983; Grant and Grant, 1983; Martínez del Río and Búrquez, 1986; Hodges, 1995). Thus, it is an ideal group in which to examine volatiles produced from hawkmoth-pollinated flowers.

Here we present results of head-space volatile collections from flowers and vegetation of species in three genera of Nyctaginaceae: Acleisanthes, Mirabilis, and 
Selinocarpus (Table 1). This is the first report of fragrance compounds for any Nyctaginaceae except Mirabilis jalapa (Heath and Manukian, 1994). In particular, we investigated within versus among species variation in fragrance compounds for 20 species of Nyctaginaceae. The prediction was that intraspecific variation would be less than interspecific variation, supporting the premise that species have distinct fragrance profiles. Further, we asked how fragrances differed within and among the three genera, and how these differences are related to pollinator affinities.

\section{Results and discussion}

Our analyses identified a wide variety of volatile compounds across all 20 species examined (Table 2), including representatives of at least seven different biosynthetic classes: monoterpenoids, sesquiterpenoids, fatty acid and amino acid-derived aliphatic compounds and lactones, benzenoids, phenylpropanoids, and nitrogen-bearing compounds [the nitrogen-bearing compounds are known to result from three different biosynthetic pathways (Radwanski and Last, 1995; Wink, 1997)]. The most commonly occurring fragrance compounds were 4,8-dimethyl-nona$1,3,7$-triene (present in all species), and trans- $\beta$-ocimene, cis-3-hexenyl acetate, and methyl salicylate (found in 18 of 20 species). There was also a preponderance of $c i s-3-$ hexenyl esters across all three genera, including both aliphatic and aromatic compounds; these may be the products of a single enzyme or a group of similar enzymes reacting with multiple substrates (see Wang and Pichersky, 1999; Dudareva and Pichersky, 2000).

\subsection{Intraspecific vs. interspecific variation}

Intraspecific variation in floral and vegetative fragrance quality (types of compounds) and quantity (amounts of compounds) was significantly lower than interspecific variation $[P<0.0001$, Wilcoxon Signed Ranks Test (SPSS Inc., 1999); mean \% dissimilarity of relative amounts of volatiles within species $=12.0($ S.D. $=6.8)$ vs. among species $=19.1($ S.D. $=7.3)]$. These results are consistent with those of Barkman et al. (1997), who found that whereas intraspecific variation was substantial in Cypripedium (Orchidaceae), it was not as great as interspecific variation. Thus, it is reasonable to conclude that each species has its own characteristic fragrance profile, which can then be compared to the fragrance profiles of other taxa (Table 2). It should be noted that the interspecific comparisons spanned a wide range of phylogenetic relationships (i.e., some species were more closely related than others; Levin, 2000), and it would be ideal to control for degree of phylogenetic relatedness (Levin, 2001). Levin (2000) has shown that Acleisanthes and Selinocarpus species together form a monophyletic group, although

Table 1

Species included in this study, and their known pollinator affiliations ${ }^{\mathrm{a}}$

\begin{tabular}{|c|c|c|}
\hline Species & Likely pollinators & Evidence \\
\hline \multicolumn{3}{|l|}{ Genus Acleisanthes A. Gray } \\
\hline A. acutifolia Standl. & Hawkmoths & Floral morphology \\
\hline A. crassifolia A. Gray & Hawkmoths & Pers. observations ${ }^{\mathrm{a}}$ \\
\hline A. longiflora A. Gray & Hawkmoths & Pers. observations; Spellenberg and Delson, 1977 \\
\hline A. obtusa (Choisy) Standl. & Hawkmoths & Pers. observations \\
\hline A. wrightii (A. Gray) Benth. \& Hook. & Hawkmoths & Pers. observations \\
\hline \multicolumn{3}{|l|}{ Genus Mirabilis L. } \\
\hline M. alipes (S. Watson) Pilz & Noctuid moths, hawkmoths & Pers. observations \\
\hline M. bigelovii A. Gray & Noctuid moths & Floral morphology \\
\hline M. greenei S. Watson & Hawkmoths & Floral morphology \\
\hline M. jalapa L. & Hawkmoths & Martínez del Río and Búrquez, 1986 \\
\hline M. longiflora L. & Hawkmoths & Grant and Grant, 1983 \\
\hline M. macfarlanei Constance \& Rollins & Bees & Barnes, 1996 \\
\hline M. multiflora (Torrey) A. Gray & Hawkmoths & Pers. observations; Cruden, 1970; Hodges, 1995 \\
\hline M. pudica Barneby & Noctuid moths & Pers. observations \\
\hline M. triflora Benth. & Hummingbirds & Pilz, 1978 \\
\hline \multicolumn{3}{|l|}{ Genus Selinocarpus A. Gray } \\
\hline S. angustifolius Torrey & Noctuid moths & Floral morphology \\
\hline S. chenopodioides A. Gray & Noctuid moths, hawkmoths, long-tongued flies & Pers. observations \\
\hline S. lanceolatus Wooton & Hawkmoths & Floral morphology \\
\hline S. parvifolius (Torrey) Standl. & Hawkmoths & Pers. observations \\
\hline S. purpusianus Heimerl & Hawkmoths & Pers. observations; Fowler and Turner, 1977 \\
\hline S. undulatus Fowler \& Turner & Noctuid moths & Floral morphology \\
\hline
\end{tabular}

a Personal observations by R. Levin include floral visitation, examination of visitors for pollen carriage, and microscopic study of stigmas for the presence of moth scales. In addition to nocturnal floral anthesis, floral morphological traits used as evidence of hawkmoth pollination included a tubular perianth $>2 \mathrm{~cm}$ long; a perianth $<2 \mathrm{~cm}$ long suggested noctuid moth pollination. 
Table 2

Mean percentage of each compound out of the total amount of volatiles found in the floral and vegetative fragrance of each species ${ }^{\mathrm{a}}$

\begin{tabular}{|c|c|c|c|c|c|c|c|c|c|c|c|c|c|c|c|c|c|c|c|c|c|}
\hline Compound $^{\mathrm{b}}$ & RT & $\begin{array}{l}\text { Aac } \\
n=1^{\mathrm{c}, \mathrm{d}}\end{array}$ & $\begin{array}{l}\text { Acr } \\
n=5^{\mathrm{d}}\end{array}$ & $\begin{array}{l}\text { Alo } \\
n=4\end{array}$ & $\begin{array}{l}\text { Aob } \\
n=5\end{array}$ & $\begin{array}{l}\text { Awr } \\
n=5\end{array}$ & $\begin{array}{l}\text { Mal } \\
n=7\end{array}$ & $\begin{array}{l}\text { Mbi } \\
n=1^{\mathrm{d}}\end{array}$ & $\begin{array}{l}\mathrm{Mgr} \\
n=6\end{array}$ & $\begin{array}{l}\mathrm{Mja} \\
n=1\end{array}$ & $\begin{array}{l}\text { Mlo } \\
n=2\end{array}$ & $\begin{array}{l}\mathrm{Mmf} \\
n=1^{\mathrm{d}}\end{array}$ & $\begin{array}{l}\mathrm{Mmu} \\
n=5\end{array}$ & $\begin{array}{l}\mathrm{Mpu} \\
n=5\end{array}$ & $\begin{array}{l}\text { Mtr } \\
n=2^{\mathrm{e}}\end{array}$ & $\begin{array}{l}\text { San } \\
n=3\end{array}$ & $\begin{array}{l}\text { Sch } \\
n=9\end{array}$ & $\begin{array}{l}\mathrm{Sla} \\
n=4\end{array}$ & $\begin{array}{l}\text { Spa } \\
n=4\end{array}$ & $\begin{array}{l}\text { Spu } \\
n=4\end{array}$ & $\begin{array}{l}\text { Sun } \\
n=4\end{array}$ \\
\hline Total amount ${ }^{\mathrm{f}}$ & & 611.06 & 56.77 & 553.90 & 99.87 & 135.84 & 9.84 & 63.73 & 0.32 & 6.66 & 7.13 & 0.19 & 4.87 & 13.92 & 0.96 & 3.30 & 174.31 & 61.44 & 415.36 & 333.46 & 1.85 \\
\hline$\%$ Floral $^{\mathrm{g}}$ & & 18.18 & 99.53 & 92.23 & 69.73 & 17.98 & 94.61 & 95.92 & 13.71 & 92.05 & 94.22 & 54.95 & 99.47 & 100.00 & 73.99 & 5.23 & 82.17 & 83.87 & 64.14 & 96.65 & 40.04 \\
\hline Total no. cmpds ${ }^{\mathrm{h}}$ & & 60 & 62 & 47 & 73 & 108 & 29 & 41 & 5 & 21 & 27 & 6 & 30 & 32 & 24 & 36 & 63 & 51 & 63 & 81 & 39 \\
\hline \# Floral cmpds ${ }^{i}$ & & 13 & 59 & 25 & 55 & 33 & 23 & 34 & 1 & 15 & 22 & 2 & 29 & 32 & 17 & 8 & 50 & 47 & 43 & 77 & 17 \\
\hline Amount floral $/ \mu \mathrm{g}$ floral mass ${ }^{\mathrm{j}}$ & & 575.54 & 26.49 & 233.74 & 102.59 & 10.4 & 8.43 & 75.78 & 0.04 & 3.84 & 5.59 & 0.13 & 2.84 & 9.75 & 3.52 & 0.90 & 236.49 & 43.59 & 352.81 & 334.63 & 3.42 \\
\hline $\begin{array}{l}\text { Monoterpenoids/irregular terpenes } \\
\alpha \text {-Pinene*k }\end{array}$ & 2.50 & 0.32 & Trace $^{1, \mathrm{~m}}$ & Trace & 0.32 & 0.06 & - & - & - & - & - & - & - & - & Trace & 0.29 & - & 0.40 & Trace & Trace & 0.57 \\
\hline Camphene* & 2.95 & 0.67 & - & Trace & 1.15 & 0.31 & - & - & - & - & - & - & - & - & - & 0.25 & - & 4.39 & - & - & 0.54 \\
\hline$\beta$-Pinene* & 4.00 & - & Trace & Trace & 1.13 & 0.02 & - & - & - & - & - & - & - & 0.03 & - & 0.45 & - & 0.56 & 0.06 & Trace & 0.14 \\
\hline Sabinene* & 4.10 & 0.15 & - & Trace & 6.50 & Trace & - & - & - & - & - & - & - & - & - & 0.29 & - & - & 0.08 & Trace & - \\
\hline 2-Carene* & 4.43 & - & - & - & - & - & - & - & - & - & 15.10 & - & - & - & - & - & - & - & - & - & - \\
\hline Phellandrene* & 5.10 & - & - & - & - & - & - & - & - & - & 0.65 & - & - & - & - & - & - & - & - & - & - \\
\hline Myrcene* & 5.14 & 1.09 & 0.59 & Trace & 1.26 & 0.31 & 0.31 & 0.08 & - & 0.58 & - & - & - & 0.30 & - & 2.80 & - & 3.09 & 0.63 & 0.73 & 1.27 \\
\hline$\alpha$-Terpinene* & 5.45 & - & - & - & Trace & 0.04 & - & - & - & - & 0.23 & - & - & - & - & - & - & - & - & - & - \\
\hline Limonene* & 5.75 & 0.54 & 0.32 & 0.24 & 15.26 & 2.29 & - & 0.14 & - & - & 3.89 & 43.35 & 1.94 & 0.88 & 4.76 & 3.14 & 0.25 & 6.87 & 0.58 & 0.07 & 9.85 \\
\hline$\beta$-Terpinene & 5.90 & - & - & - & - & - & - & - & - & - & 9.91 & - & - & - & - & 1.39 & - & - & - & - & - \\
\hline cis- $\beta$-Ocimene* & 6.53 & 0.16 & 0.18 & Trace & 0.13 & 0.05 & 1.05 & 0.13 & - & 1.31 & 0.83 & - & - & 0.02 & - & 4.68 & 0.10 & 1.52 & 0.33 & 1.05 & 1.41 \\
\hline trans- $\beta$-Ocimene* & 6.97 & 1.50 & 10.08 & 0.43 & 5.39 & 0.82 & 60.79 & 7.21 & - & 38.10 & 3.44 & - & 1.72 & 2.24 & 1.30 & 12.14 & 0.76 & 5.87 & 0.76 & 57.81 & 3.45 \\
\hline$\alpha$-Terpinolene* & 7.31 & 0.06 & Trace & - & 0.28 & 0.06 & - & 0.01 & - & - & 0.24 & - & Trace & 0.08 & - & - & - & 0.94 & 0.15 & Trace & - \\
\hline 6-Methyl-5-hepten-2-one & 8.27 & - & - & 6.16 & 4.99 & - & 1.34 & - & 4.36 & - & - & 0.52 & - & 0.76 & - & - & 0.58 & 3.67 & 0.72 & 0.10 & 5.53 \\
\hline$\alpha$-Pinene epoxides & $\begin{array}{l}8.57 \\
9.16\end{array}$ & - & - & - & - & - & - & - & - & - & - & - & - & - & - & - & - & - & - & 0.14 & - \\
\hline 2,7-Methyl Octadiene & 8.63 & - & - & - & - & 0.03 & - & - & - & - & - & - & - & - & - & - & - & - & - & - & - \\
\hline Allo-Ocimene* & 8.80 & - & Trace & - & 0.02 & Trace & - & 0.02 & - & Trace & - & - & - & - & - & 0.52 & - & - & - & 0.16 & - \\
\hline Cinerone/piperitenone & $\begin{array}{l}9.26 \\
9.9\end{array}$ & - & - & - & - & - & - & - & - & - & - & - & - & - & - & - & - & - & - & 0.15 & - \\
\hline Furanoid linalool oxides* & $\begin{array}{l}9.8 \\
10.3\end{array}$ & - & - & - & - & 0.03 & 7.18 & - & - & - & - & - & - & 4.16 & - & - & - & - & - & - & 2.28 \\
\hline Menthatriene & 9.91 & - & - & - & - & - & 0.61 & - & - & - & - & - & - & - & - & - & - & Trace & - & 0.17 & - \\
\hline Santene* & 10.68 & - & - & - & - & Trace & - & - & - & - & - & - & - & - & - & - & - & - & - & - & - \\
\hline Octadiene & 11.07 & - & - & - & - & - & 0.47 & - & - & - & - & - & - & - & - & - & - & - & - & 0.07 & - \\
\hline Linalool* & 11.25 & 0.19 & 0.32 & 0.51 & 0.80 & 2.52 & 0.66 & 0.38 & - & - & - & - & 0.53 & 2.00 & 8.55 & 1.85 & 0.92 & 3.93 & 1.21 & 0.30 & 3.72 \\
\hline Isocitronellen & 11.67 & - & - & - & - & - & - & - & - & - & - & - & - & - & - & - & - & 0.31 & 0.76 & - & - \\
\hline 4-Terpineol* & 11.95 & - & - & - & - & - & - & - & - & - & - & - & - & - & - & - & - & - & - & - & 7.93 \\
\hline cis-Citral* & 12.76 & - & Trace & - & - & 0.03 & - & - & - & - & Trace & - & - & - & - & - & - & - & - & 0.08 & - \\
\hline Limonene diepoxides & $13-13.9$ & - & - & - & 0.08 & Trace & - & - & - & - & - & - & - & - & 0.80 & 0.91 & - & - & - & 0.07 & - \\
\hline Eucarvone* & 13.44 & - & - & - & - & - & - & - & - & - & - & - & - & - & - & - & - & - & - & 0.11 & - \\
\hline$(-)$-Verbenone* & 13.54 & - & - & - & - & - & - & - & - & - & - & - & - & - & - & - & - & - & - & 0.73 & - \\
\hline trans-Citral* & 13.61 & - & - & - & - & 0.06 & - & - & - & - & - & - & - & - & - & - & - & - & - & - & - \\
\hline Pyranoid linalool oxides* & $\begin{array}{l}13.7 \\
13.9\end{array}$ & - & - & - & - & - & 10.60 & - & - & - & - & - & 0.68 & 20.04 & - & - & - & - & - & - & 1.70 \\
\hline Nerol* & 14.3 & 2.72 & - & - & - & - & - & 0.03 & - & - & 0.49 & - & 0.35 & - & 3.59 & 8.27 & 7.02 & - & - & - & 2.81 \\
\hline$\alpha$-Ionone* & 14.46 & - & - & - & - & - & - & - & - & - & - & - & - & - & - & - & - & - & - & 0.08 & - \\
\hline Carveol* & 14.57 & - & 0.03 & - & - & 0.05 & - & 0.05 & - & - & - & - & - & - & - & - & 0.09 & - & - & 0.04 & - \\
\hline
\end{tabular}




\begin{tabular}{|c|c|c|c|c|c|c|c|c|c|c|c|c|c|c|c|c|c|c|c|c|c|}
\hline Compound $^{\mathrm{b}}$ & RT & $\begin{array}{l}\text { Aac } \\
n=1^{\mathrm{c}, \mathrm{d}}\end{array}$ & $\begin{array}{l}\text { Acr } \\
n=5^{\mathrm{d}}\end{array}$ & $\begin{array}{l}\text { Alo } \\
n=4\end{array}$ & $\begin{array}{l}\text { Aob } \\
n=5\end{array}$ & $\begin{array}{l}\text { Awr } \\
n=5\end{array}$ & $\begin{array}{l}\text { Mal } \\
n=7\end{array}$ & $\begin{array}{l}\text { Mbi } \\
n=1^{\mathrm{d}}\end{array}$ & $\begin{array}{l}\text { Mgr } \\
n=6\end{array}$ & $\begin{array}{l}\mathrm{Mja} \\
n=1\end{array}$ & $\begin{array}{l}\text { Mlo } \\
n=2\end{array}$ & $\begin{array}{l}\mathrm{Mmf} \\
n=1^{\mathrm{d}}\end{array}$ & $\begin{array}{l}\mathrm{Mmu} \\
n=5\end{array}$ & $\begin{array}{l}\mathrm{Mpu} \\
n=5\end{array}$ & $\begin{array}{l}\mathrm{Mtr} \\
n=2^{\mathrm{e}}\end{array}$ & $\begin{array}{l}\text { San } \\
n=3\end{array}$ & $\begin{array}{l}\text { Sch } \\
n=9\end{array}$ & $\begin{array}{l}\mathrm{Sla} \\
n=4\end{array}$ & $\begin{array}{l}\text { Spa } \\
n=4\end{array}$ & $\begin{array}{l}\text { Spu } \\
n=4\end{array}$ & $\begin{array}{l}\text { Sun } \\
n=4\end{array}$ \\
\hline $\begin{array}{l}\text { Thujol } \\
\text { Unknowns with } 150 \text { as mol. ion }\end{array}$ & 16.38 & - & 0.15 & - & - & - & - & - & - & - & - & - & - & - & - & - & - & - & - & 0.05 & - \\
\hline $150,135,43,67,55^{\mathrm{n}}$ & 7.76 & _- & - & - & _- & - & - & - & - & - & _- & - & - & - & _- & - & - & Trace & Trace & 0.02 & - \\
\hline $150,39,95,77,91$ & 11.07 & - & - & - & - & - & - & - & - & - & - & - & - & - & - & - & - & - & - & 0.08 & - \\
\hline $150,39,41,91,79,107,135$ & 17.18 & - & - & - & - & 0.16 & - & - & - & - & - & - & - & - & - & - & - & - & - & 0.36 & - \\
\hline $150,41,107,53,79,135,91$ & 19.18 & - & - & - & - & 0.20 & - & - & - & - & - & - & - & - & - & - & - & - & - & - & - \\
\hline Other unknowns & & 1.37 & 0.10 & 0.09 & 1.34 & 0.38 & 1.41 & 0.02 & - & 3.12 & 0.46 & - & - & 0.05 & Trace & 0.72 & Trace & 1.65 & 0.31 & 1.43 & 10.83 \\
\hline Total $\%$ & & 8.78 & 11.76 & 7.44 & 38.65 & 7.42 & 84.41 & 8.07 & 4.36 & 43.11 & 35.25 & 43.87 & 5.23 & 30.56 & 19.03 & 37.71 & 9.73 & 33.21 & 5.57 & 63.83 & 52.02 \\
\hline \multicolumn{22}{|l|}{ Sesquiterpenoids } \\
\hline 4,8-dimethyl-nona-1,3,7-triene* & 7.76 & 0.84 & 0.47 & 0.20 & 0.68 & Trace & 5.03 & 23.84 & 13.71 & 0.81 & 4.41 & 11.60 & 5.18 & 12.25 & 2.93 & 15.23 & 1.29 & 10.37 & 0.53 & 0.02 & 2.01 \\
\hline Other likely homoterpenes & $\begin{array}{l}10.47 \\
14.10 \\
14.41\end{array}$ & - & 0.14 & 0.02 & 0.06 & 0.21 & 0.74 & - & - & - & - & - & - & 0.21 & 1.04 & 0.93 & Trace & 0.14 & - & - & - \\
\hline$\alpha$-Cubebene* & 9.95 & 0.02 & - & - & - & Trace & - & - & - & - & - & - & - & - & - & - & - & - & - & - & - \\
\hline$\delta$-Elemene* & 10.23 & 0.05 & - & 0.04 & Trace & 0.12 & - & - & - & - & - & - & - & - & - & - & 0.15 & 0.44 & 0.08 & - & - \\
\hline$\alpha$-Copaene* & 10.54 & 0.16 & 0.03 & - & Trace & 0.12 & - & - & - & - & 0.31 & - & - & - & - & - & - & - & - & - & - \\
\hline$\beta$-Bourbonene* & $\begin{array}{l}10.84 \\
10.92\end{array}$ & 0.74 & - & - & - & 0.51 & - & - & - & - & - & - & - & - & - & 2.42 & - & - & - & - & Trace \\
\hline$\alpha$-Gurjunene & 11.06 & - & - & - & - & 0.02 & - & - & - & - & - & - & - & - & - & Trace & - & - & - & - & - \\
\hline$\beta$-Cubebene* & 11.17 & 0.42 & 0.01 & - & - & 0.03 & - & 0.01 & - & - & - & - & - & - & - & - & - & - & - & - & - \\
\hline$\alpha$-Bergamotene* & 11.60 & - & 0.04 & - & 1.42 & - & 0.29 & - & - & - & 0.65 & - & - & - & - & - & 0.20 & - & - & - & - \\
\hline$\beta$-Elemene* & 11.70 & 6.86 & 0.04 & - & - & 12.74 & - & 0.30 & - & - & - & - & - & - & - & - & - & - & - & 0.04 & - \\
\hline Caryophyllene* & 11.98 & 7.36 & 3.52 & - & 1.52 & 4.35 & - & 24.85 & - & - & 0.93 & - & 0.40 & - & - & 9.84 & 0.73 & 2.19 & 0.41 & - & - \\
\hline Chamigrene & 12.30 & Trace & - & - & - & - & - & - & - & - & Trace & - & - & - & - & 1.07 & - & Trace & 0.08 & - & 0.47 \\
\hline$\beta$-Selinene & 12.48 & 0.63 & - & - & - & 0.17 & - & - & - & - & - & - & - & - & - & - & - & - & - & Trace & - \\
\hline$\beta$-Farnesene* & $\begin{array}{l}12.7 \\
13.1\end{array}$ & 1.06 & Trace & 0.26 & 1.36 & 0.05 & - & 0.09 & - & - & - & - & Trace & - & - & - & 0.12 & - & - & - & - \\
\hline$\alpha$-Humulene* & 12.9 & 4.11 & 0.22 & - & 1.11 & 11.73 & - & 4.38 & - & - & Trace & - & - & - & - & 2.37 & 0.14 & - & 0.14 & - & - \\
\hline$\beta$-Cadinene* & 13.11 & - & - & - & - & 0.07 & - & - & - & - & - & - & - & - & - & - & - & - & - & - & - \\
\hline Germacrene $\mathrm{D}^{*}$ & $\begin{array}{l}13.4 \\
13.7\end{array}$ & 12.27 & - & Trace & 2.34 & 1.57 & - & - & - & - & Trace & - & - & - & - & 4.11 & 0.01 & 1.26 & 0.18 & - & - \\
\hline$\alpha$-Farnesene* & $\begin{array}{l}13.48- \\
13.80\end{array}$ & - & 2.00 & 0.52 & 18.95 & 0.07 & 0.42 & 0.02 & - & 37.03 & 4.37 & - & - & 0.97 & - & - & 0.53 & - & 0.09 & 2.63 & 0.52 \\
\hline$\delta$-Guaiene* & 13.52 & 7.72 & - & - & - & - & - & - & - & - & - & - & - & - & - & - & - & - & - & - & - \\
\hline Junipene & 13.54 & 0.70 & - & - & - & 2.99 & - & - & - & - & - & - & - & - & - & - & 0.49 & - & - & - & - \\
\hline$\gamma$-Cadinene* & 14.02 & 8.01 & - & - & Trace & 6.83 & - & - & - & - & - & - & - & - & - & - & 0.01 & - & 0.06 & - & - \\
\hline$\alpha$-Muurolene* & 14.33 & 0.58 & - & - & - & 0.22 & - & - & - & - & - & - & - & - & - & - & - & - & - & - & - \\
\hline Calamenene* & 14.79 & 0.78 & - & - & - & 0.16 & - & - & - & - & - & - & - & - & - & - & - & - & - & - & - \\
\hline Geranyl acetone* & 14.95 & - & - & 0.45 & - & - & 0.10 & - & - & - & - & - & 0.45 & 0.35 & 1.28 & 1.38 & 0.63 & 1.02 & 0.36 & 0.08 & 2.46 \\
\hline Caryophyllene oxides* & $\begin{array}{l}16.2 \\
16.5\end{array}$ & 6.18 & 0.12 & - & Trace & 3.75 & - & 2.39 & - & - & - & - & - & - & - & - & 0.23 & - & - & - & - \\
\hline Nerolidol* & $\begin{array}{l}16.46- \\
16.87\end{array}$ & 2.55 & 0.14 & 80.46 & 1.06 & 0.28 & - & 0.05 & - & 0.21 & - & - & - & - & - & - & 0.14 & - & Trace & 0.01 & - \\
\hline 1,5-Cyclo-undecadiene ${ }^{\circ}$ & 16.69 & - & - & - & - & 0.06 & - & - & - & - & - & - & - & - & - & - & - & - & - & - & - \\
\hline 12-Oxabicyclo dodecadiene $\mathrm{p}^{\mathrm{P}}$ & 17.04 & 0.57 & 0.01 & - & - & 0.98 & - & 0.58 & - & - & - & - & - & - & - & - & - & - & - & - & - \\
\hline
\end{tabular}


Table 2 (continued)

\begin{tabular}{|c|c|c|c|c|c|c|c|c|c|c|c|c|c|c|c|c|c|c|c|c|c|}
\hline Compound $^{\mathrm{b}}$ & $\mathrm{RT}$ & $\begin{array}{l}\mathrm{Aac} \\
n=1^{\mathrm{c}, \mathrm{d}}\end{array}$ & $\begin{array}{l}\text { Acr } \\
n=5^{\text {d }}\end{array}$ & $\begin{array}{l}\text { Alo } \\
n=4\end{array}$ & $\begin{array}{l}\text { Aob } \\
n=5\end{array}$ & $\begin{array}{l}\text { Awr } \\
n=5\end{array}$ & $\begin{array}{l}\text { Mal } \\
n=7\end{array}$ & $\begin{array}{l}\mathrm{Mbi} \\
n=1^{\mathrm{d}}\end{array}$ & $\begin{array}{l}\text { Mgr } \\
n=6\end{array}$ & $\begin{array}{l}\mathrm{Mja} \\
n=1\end{array}$ & $\begin{array}{l}\text { Mlo } \\
n=2\end{array}$ & $\begin{array}{l}\mathrm{Mmf} \\
n=1^{\mathrm{d}}\end{array}$ & $\begin{array}{l}\text { Mmu } \\
n=5\end{array}$ & $\begin{array}{l}\mathrm{Mpu} \\
n=5\end{array}$ & $\begin{array}{l}\mathrm{Mtr} \\
n=2^{\mathrm{e}}\end{array}$ & $\begin{array}{l}\text { San } \\
n=3\end{array}$ & $\begin{array}{l}\text { Sch } \\
n=9\end{array}$ & $\begin{array}{l}\text { Sla } \\
n=4\end{array}$ & $\begin{array}{l}\text { Spa } \\
n=4\end{array}$ & $\begin{array}{l}\text { Spu } \\
n=4\end{array}$ & $\begin{array}{l}\text { Sun } \\
n=4\end{array}$ \\
\hline Germacrone & 17.33 & - & - & - & - & 0.05 & - & - & - & - & - & - & - & - & - & - & - & - & - & - & - \\
\hline Carotol & 17.60 & - & - & - & - & 0.07 & - & - & - & - & - & - & - & - & - & - & - & - & - & - & - \\
\hline Spathulenol & 17.78 & - & - & - & - & 0.11 & - & - & - & - & - & - & - & - & - & - & - & - & - & - & - \\
\hline Aromadendrene epoxide & 17.99 & - & - & - & - & 0.22 & - & - & - & - & - & - & - & - & - & - & - & - & - & - & - \\
\hline$\delta$-Cadinol & 18.28 & - & - & - & - & 9.14 & - & - & - & - & - & - & - & - & - & - & - & - & - & - & - \\
\hline Guaiol & 18.34 & - & - & - & - & 0.22 & - & - & - & - & - & - & - & - & - & - & - & - & - & - & - \\
\hline Aristolene & 18.41 & - & - & - & - & 0.35 & - & - & - & - & - & - & - & - & - & - & - & - & - & - & - \\
\hline$\alpha, \beta$-Eudesmol & $\begin{array}{l}18.73 \\
18.86\end{array}$ & 2.11 & - & - & - & 10.41 & - & - & - & - & - & - & - & - & - & - & - & - & - & - & - \\
\hline Farnesol* & $\begin{array}{l}18.9- \\
19.8\end{array}$ & 0.64 & 0.19 & 0.95 & 3.02 & 3.55 & - & - & - & - & - & - & - & - & - & - & - & - & - & - & - \\
\hline Nerolidol epoxyacetate & 21.58 & - & - & - & - & 0.12 & - & - & - & - & - & - & - & - & - & - & - & - & - & - & - \\
\hline \multicolumn{22}{|l|}{ Unknown sesquiterpenoids } \\
\hline $204,41,107,81,91,67,121$ & 13.85 & - & 0.05 & Trace & - & 0.09 & - & 0.03 & - & - & - & - & - & - & - & 2.34 & - & - & - & - & 0.30 \\
\hline $222,43,161,55,81,91,207,105,119$ & $\begin{array}{l}15.36 \\
15.75\end{array}$ & 1.27 & $\mathbf{0 . 0 8}$ & - & 0.08 & 0.15 & - & Trace & - & 0.70 & - & - & - & - & - & 0.44 & - & - & - & 0.03 & - \\
\hline $222,81,41,55,93,121,69,136,109$ & 15.87 & - & Trace & - & 0.31 & 1.02 & - & - & - & - & - & - & - & - & 1.55 & - & - & - & 0.12 & 0.04 & - \\
\hline $222,41,81,55,93,69,109,161,121,207$ & 15.90 & - & - & - & - & 0.66 & - & - & - & - & - & - & - & - & - & - & - & - & - & - & - \\
\hline $220,43,79,93,55,69,109,121$ & 16.50 & - & - & - & - & 2.55 & - & - & - & - & - & - & - & - & - & - & - & - & - & - & - \\
\hline $222,81,43,161,105,71,91,55,119,207$ & 17.07 & 0.41 & - & - & - & 0.44 & - & - & - & - & - & - & - & - & - & - & - & - & - & - & - \\
\hline $222,41,119,161,55,179,105,81,95,204$ & 17.16 & - & - & - & - & 0.33 & - & - & - & - & - & - & - & - & - & - & - & - & - & - & - \\
\hline Other unknowns & & 5.10 & - & - & Trace & 2.60 & - & Trace & - & - & - & - & - & - & - & - & - & - & - & - & - \\
\hline Total \% & & 71.14 & 7.08 & 82.90 & 31.92 & 79.13 & 6.58 & 56.55 & 13.71 & 38.75 & 10.69 & 11.60 & 6.03 & 13.78 & 6.80 & 40.13 & 4.66 & 15.42 & 2.04 & 2.86 & 5.77 \\
\hline \multicolumn{22}{|l|}{ Aliphatic compounds } \\
\hline 2-Hexenal* & 6.21 & - & - & 0.05 & 0.24 & - & - & - & - & - & - & - & - & - & - & - & - & 0.44 & 0.70 & - & - \\
\hline cis-3-Hexenyl acetate* & 7.95 & 2.16 & 9.52 & 3.27 & 2.00 & 5.73 & 3.33 & 29.00 & 52.43 & 3.44 & - & - & 6.49 & 31.22 & 5.51 & 11.66 & 9.28 & 7.71 & 15.04 & 1.08 & 11.19 \\
\hline trans-2-Hexenyl acetate* & 8.18 & 0.01 & 0.04 & - & Trace & 0.01 & - & 0.43 & - & - & - & - & - & - & - & - & 0.76 & - & - & - & - \\
\hline 1-Hexanol* & 8.42 & - & 0.23 & 0.51 & 1.06 & 0.05 & Trace & 0.13 & 11.35 & - & - & 21.65 & 1.63 & 0.34 & - & - & 0.93 & 3.26 & 0.74 & 0.08 & 1.91 \\
\hline 1-Octen-3-ol acetate* & 8.87 & - & - & - & - & 0.25 & - & 0.37 & - & - & - & - & - & - & - & - & - & - & - & - & - \\
\hline cis-3-Hexen-1-ol* & 8.95 & 0.22 & 5.08 & 1.03 & 0.86 & 0.91 & 1.68 & 3.21 & 18.15 & 0.79 & 0.94 & 19.39 & 12.89 & 12.95 & 17.83 & 1.44 & 3.43 & 7.49 & 13.96 & 0.34 & 2.90 \\
\hline cis-3-Hexenyl propionate* & 8.97 & - & - & 0.03 & - & - & - & - & - & - & - & - & - & - & - & - & - & 0.29 & 0.46 & - & - \\
\hline Butanoic acid, 3-hexenyl ester & $\begin{array}{l}9.04 \\
10.1\end{array}$ & - & 0.13 & 0.20 & - & 0.02 & - & 0.04 & - & - & - & - & 1.35 & 1.55 & 12.64 & - & 0.05 & 3.20 & 4.85 & 0.16 & - \\
\hline trans-2-Hexen-1-ol* & 9.37 & - & $\mathbf{0 . 0 3}$ & - & - & Trace & 0.19 & 0.26 & - & - & - & - & 0.76 & 0.21 & - & - & 0.37 & - & - & - & - \\
\hline 7-Octen-4-ol & 9.89 & 0.31 & 0.13 & Trace & 6.64 & 0.36 & - & 0.30 & - & - & 0.25 & - & - & - & 7.74 & - & 1.68 & 0.62 & - & - & 0.55 \\
\hline cis-3-Hexenyl 2-methyl butanoate & $\begin{array}{l}10.27 \\
10.48\end{array}$ & - & 0.09 & 0.10 & Trace & 0.04 & - & 0.11 & - & 0.43 & - & - & 1.34 & 2.74 & 11.02 & - & 0.12 & 2.19 & 5.16 & 0.11 & - \\
\hline 2-Nonenal & 11.14 & - & Trace & - & 0.53 & - & - & - & - & - & - & - & - & - & 1.77 & - & - & Trace & 0.01 & - & 1.68 \\
\hline cis-3-Hexenyl 3-methyl butanoate & 11.40 & - & Trace & - & - & - & - & - & - & - & - & - & - & - & - & 0.20 & - & - & 0.17 & - & - \\
\hline Butanoic acid, 2-hexenyl ester & 11.76 & - & - & 2.07 & - & - & - & - & - & - & - & - & - & - & - & - & - & - & - & - & - \\
\hline 2,6-Nonadienal & 11.80 & - & - & - & - & - & - & - & - & - & - & - & - & - & - & - & - & - & - & - & 5.48 \\
\hline Hexanoic acid, 3-hexenyl ester & $\begin{array}{l}12.07, \\
12.65\end{array}$ & - & 0.02 & - & - & Trace & - & - & - & - & - & - & - & - & - & - & - & 0.92 & 2.26 & - & - \\
\hline Isobutyl tiglate & 12.22 & - & - & - & - & - & - & - & - & - & - & - & - & - & - & - & - & - & 0.26 & - & - \\
\hline
\end{tabular}




\begin{tabular}{|c|c|c|c|c|c|c|c|c|c|c|c|c|c|c|c|c|c|c|c|c|c|}
\hline Compound $^{\mathrm{b}}$ & RT & $\begin{array}{l}\text { Aac } \\
n=1^{\mathrm{c}, \mathrm{d}}\end{array}$ & $\begin{array}{l}\text { Acr } \\
n=5^{\text {d }}\end{array}$ & $\begin{array}{l}\text { Alo } \\
n=4\end{array}$ & $\begin{array}{l}\text { Aob } \\
n=5\end{array}$ & $\begin{array}{l}\text { Awr } \\
n=5\end{array}$ & $\begin{array}{l}\text { Mal } \\
n=7\end{array}$ & $\begin{array}{l}\mathrm{Mbi} \\
n=1^{\mathrm{d}}\end{array}$ & $\begin{array}{l}\mathrm{Mgr} \\
n=6\end{array}$ & $\begin{array}{l}\mathrm{Mja} \\
n=1\end{array}$ & $\begin{array}{l}\text { Mlo } \\
n=2\end{array}$ & $\begin{array}{l}\mathrm{Mmf} \\
n=1^{\mathrm{d}}\end{array}$ & $\begin{array}{l}\mathrm{Mmu} \\
n=5\end{array}$ & $\begin{array}{l}\mathrm{Mpu} \\
n=5\end{array}$ & $\begin{array}{l}\text { Mtr } \\
n=2^{\mathrm{e}}\end{array}$ & $\begin{array}{l}\text { San } \\
n=3\end{array}$ & $\begin{array}{l}\text { Sch } \\
n=9\end{array}$ & $\begin{array}{l}\text { Sla } \\
n=4\end{array}$ & $\begin{array}{l}\text { Spa } \\
n=4\end{array}$ & $\begin{array}{l}\text { Spu } \\
n=4\end{array}$ & $\begin{array}{l}\text { Sun } \\
n=4\end{array}$ \\
\hline cis-3-Hexenyl tiglate & 12.83 & - & 0.02 & - & - & Trace & - & 0.05 & - & - & - & - & 0.32 & - & - & - & 0.29 & 5.22 & 31.11 & 0.13 & - \\
\hline Heptanoic acid, 3-hexenyl ester & 13.42 & - & Trace & - & - & - & - & - & - & - & - & - & - & - & - & - & - & 0.25 & 0.38 & - & - \\
\hline Hexyl butanoate & 15.32 & - & 0.14 & - & 0.07 & - & - & - & - & - & - & - & - & - & - & - & - & - & - & - & 4.04 \\
\hline cis-Jasmone* & 16.1 & 0.14 & 16.83 & Trace & Trace & 0.84 & - & - & _- & 1.32 & 4.09 & _- & - & - & - & - & 0.45 & - & 0.37 & 24.71 & - \\
\hline Benzyl Tiglate & 17.56 & - & - & - & - & - & - & - & - & - & - & - & - & - & - & - & 0.49 & - & 0.09 & - & - \\
\hline Methyl caprate & 18.55 & - & - & - & 0.18 & - & - & - & - & - & - & - & - & - & - & - & - & - & - & - & - \\
\hline Unknowns & & Trace & 0.14 & 0.16 & 0.29 & 0.04 & - & 0.22 & - & - & - & - & 0.53 & - & - & - & 0.54 & Trace & 0.49 & 1.20 & 0.39 \\
\hline Total $\%$ & & 2.84 & 32.40 & 7.42 & 11.88 & 8.25 & 5.19 & 34.11 & 81.93 & 5.97 & 5.28 & 41.04 & 25.31 & 49.02 & 56.50 & 13.31 & 18.37 & 31.60 & 76.04 & 27.80 & 28.15 \\
\hline \multicolumn{22}{|l|}{ Benzenoids } \\
\hline Anisole* & 8.37 & 0.13 & 0.17 & - & - & 0.13 & - & - & - & - & 2.38 & - & - & - & - & 3.40 & 0.78 & - & - & - & - \\
\hline Benzyl methyl ether* & 9.10 & - & - & - & - & - & - & - & - & - & - & - & - & - & - & - & 0.48 & - & - & - & - \\
\hline Other benzyl ethers & $\begin{array}{l}10.75 \\
13.34\end{array}$ & - & - & - & - & - & - & - & - & - & - & - & - & - & - & - & - & - & - & 0.47 & - \\
\hline Benzaldehyde* & 11.05 & - & - & 0.18 & 1.67 & - & 0.54 & - & - & - & - & - & 0.91 & 0.37 & - & - & 2.32 & - & 0.30 & - & 5.25 \\
\hline Methyl benzoate* & 12.33 & 0.18 & 44.44 & 0.62 & 8.05 & - & - & Trace & - & 0.44 & - & - & 44.53 & - & 0.75 & 1.00 & 35.90 & 1.93 & 0.36 & Trace & 1.16 \\
\hline Phenyl acetaldehyde* & 12.54 & - & - & 0.04 & 0.05 & - & 1.74 & - & - & - & - & - & - & 1.67 & - & - & 0.09 & 0.24 & 0.05 & - & - \\
\hline Ethyl benzoate* & 12.9 & - & 0.16 & - & - & - & - & - & - & - & - & - & 0.09 & - & - & - & 0.07 & 0.05 & - & - & - \\
\hline Salicylaldehyde & 13.00 & - & - & 0.01 & Trace & - & - & - & - & - & - & - & - & - & - & - & 0.17 & 0.65 & Trace & Trace & - \\
\hline Benzyl acetate* & 13.59 & - & - & 0.07 & 0.92 & - & 0.10 & 0.09 & - & 5.31 & - & - & 1.27 & 0.33 & 1.20 & 0.63 & 0.45 & - & 0.03 & 0.03 & - \\
\hline Propyl benzoate* & 13.95 & - & - & - & - & - & - & - & - & - & - & - & - & - & - & - & 0.03 & - & - & - & - \\
\hline Methyl salicylate* & 14.20 & 2.54 & 0.19 & 0.18 & 0.83 & 0.06 & 0.50 & 0.86 & - & 0.57 & 0.03 & - & 2.81 & 0.21 & 8.14 & 1.59 & 2.80 & 0.92 & 0.23 & 0.05 & 1.20 \\
\hline Isobutyl benzoate* & 14.32 & - & 0.12 & - & Trace & - & - & - & - & - & - & - & - & - & - & - & 0.16 & Trace & 0.02 & 0.11 & - \\
\hline Phenethyl acetate* & 14.60 & - & - & Trace & - & - & 0.16 & - & - & - & - & - & - & 0.18 & - & Trace & - & - & - & - & Trace \\
\hline Butyl benzoate* & 15.14 & - & 0.09 & - & 0.03 & 0.04 & - & - & - & - & - & - & - & - & - & - & 0.33 & 0.45 & 0.08 & 0.05 & - \\
\hline Benzyl butanoate* & 15.18 & - & - & - & Trace & 0.02 & - & - & - & 0.27 & - & - & - & - & - & - & 0.06 & - & - & Trace & - \\
\hline Benzyl alcohol* & 15.25 & - & - & 0.15 & 1.02 & - & 0.03 & - & - & 1.67 & - & 3.48 & 1.54 & 0.17 & 4.14 & 2.22 & 1.28 & 0.72 & 0.10 & 0.09 & 4.13 \\
\hline Benzyl 3-methylbutanoate & 15.42 & - & - & - & 0.01 & 0.20 & - & - & - & - & - & - & - & - & - & - & 0.21 & - & - & 0.03 & - \\
\hline Isopropyl salicylate & 15.47 & - & - & - & - & - & - & - & - & - & - & - & - & - & - & - & 0.05 & - & - & - & - \\
\hline 2-Phenylethanol* & 15.54 & - & Trace & 0.07 & 0.12 & Trace & 0.43 & - & - & - & - & - & - & 0.93 & - & - & 0.26 & 0.23 & 0.27 & 0.07 & 0.47 \\
\hline Pentyl benzoate* & 15.68 & 0.42 & 0.08 & - & 0.02 & 0.16 & - & - & - & - & - & - & - & - & - & - & 0.25 & - & 0.10 & 0.12 & - \\
\hline Hexyl benzoate* & 16.26 & - & 0.01 & - & - & - & - & - & - & - & - & - & Trace & - & - & - & 0.62 & - & 0.04 & 0.01 & - \\
\hline Tolyl/Allyl benzoate & 16.89 & - & 0.21 & - & Trace & Trace & - & - & - & - & - & - & - & - & - & - & 0.15 & Trace & 0.21 & 0.12 & - \\
\hline Cyclohexyl benzoate & 17.23 & - & 0.36 & - & 0.04 & Trace & - & 0.13 & - & - & - & - & 1.31 & - & Trace & - & 0.70 & - & 0.07 & 0.25 & - \\
\hline cis-3-Hexenyl benzoate* & $\begin{array}{l}17.62- \\
17.96\end{array}$ & 1.72 & 2.21 & 0.08 & 0.23 & 0.25 & - & 0.14 & - & 0.27 & - & - & 7.87 & 0.28 & 2.14 & - & 3.09 & 1.13 & 3.73 & 1.88 & - \\
\hline Phenethyl benzoate & 17.80 & - & - & - & - & - & - & - & - & - & - & - & - & 0.83 & - & - & - & - & - & - & - \\
\hline Benzyl benzoate* & 22.30 & - & 0.21 & Trace & 4.03 & 0.21 & - & - & - & 3.63 & - & _- & 1.43 & 0.14 & 1.30 & - & 7.41 & - & - & 0.32 & - \\
\hline Unknown: $105,77,41,51,67$ & 15.20 & - & - & - & - & - & - & - & - & - & - & - & - & - & - & - & - & 0.13 & 0.12 & - & - \\
\hline Other Unknowns & & - & Trace & - & 0.05 & - & - & - & - & - & - & - & Trace & - & - & - & - & - & - & - & - \\
\hline Total $\%$ & & 4.99 & 48.26 & 1.41 & 17.07 & 1.09 & 3.51 & 1.22 & - & 12.17 & 2.40 & 3.48 & 61.75 & 5.12 & 17.68 & 8.85 & 57.67 & 6.45 & 5.71 & 3.62 & 12.22 \\
\hline \multicolumn{22}{|l|}{ Phenylpropanoids } \\
\hline Eugenol* & 18.20 & 2.53 & - & - & - & - & - & - & - & - & - & - & - & - & - & - & 0.05 & - & - & - & - \\
\hline Eugenol acetate/Isoeugenol* & 19.07 & - & - & - & - & 0.08 & - & - & - & - & - & - & - & - & - & - & - & - & - & - & - \\
\hline Isoeugenol* & 19.88 & 6.55 & - & - & - & 2.91 & - & - & - & - & - & - & - & - & - & - & Trace & - & - & - & - \\
\hline
\end{tabular}


Table 2 (continued)

\begin{tabular}{|c|c|c|c|c|c|c|c|c|c|c|c|c|c|c|c|c|c|c|c|c|c|}
\hline Compound $^{\mathrm{b}}$ & $\mathrm{RT}$ & $\begin{array}{l}\mathrm{Aac} \\
n=1^{\mathrm{c}, \mathrm{d}}\end{array}$ & $\begin{array}{l}\text { Acr } \\
n=5^{\text {d }}\end{array}$ & $\begin{array}{l}\text { Alo } \\
n=4\end{array}$ & $\begin{array}{l}\text { Aob } \\
n=5\end{array}$ & $\begin{array}{l}\text { Awr } \\
n=5\end{array}$ & $\begin{array}{l}\text { Mal } \\
n=7\end{array}$ & $\begin{array}{l}\text { Mbi } \\
n=1^{\mathrm{d}}\end{array}$ & $\begin{array}{l}\mathrm{Mgr} \\
n=6\end{array}$ & $\begin{array}{l}\mathrm{Mja} \\
n=1\end{array}$ & $\begin{array}{l}\text { Mlo } \\
n=2\end{array}$ & $\begin{array}{l}\mathrm{Mmf} \\
n=1^{\mathrm{d}}\end{array}$ & $\begin{array}{l}\mathrm{Mmu} \\
n=5\end{array}$ & $\begin{array}{l}\text { Mpu } \\
n=5\end{array}$ & $\begin{array}{l}\mathrm{Mtr} \\
n=2^{\mathrm{e}}\end{array}$ & $\begin{array}{l}\text { San } \\
n=3\end{array}$ & $\begin{array}{l}\text { Sch } \\
n=9\end{array}$ & $\begin{array}{l}\text { Sla } \\
n=4\end{array}$ & $\begin{array}{l}\text { Spa } \\
n=4\end{array}$ & $\begin{array}{l}\mathrm{Spu} \\
n=4\end{array}$ & $\begin{array}{l}\text { Sun } \\
n=4\end{array}$ \\
\hline Vanillin* & 21.70 & - & - & - & - & 0.20 & - & - & - & - & - & - & - & - & - & - & - & - & - & - & - \\
\hline Vanillyl methylketone & 22.50 & - & - & - & - & 0.19 & - & - & - & - & - & - & - & - & - & - & - & - & - & - & - \\
\hline Total $\%$ & & 9.07 & - & - & - & 3.38 & - & - & - & - & - & - & - & - & - & - & 0.05 & - & - & - & - \\
\hline \multicolumn{22}{|l|}{ Lactones } \\
\hline 4,4-Dimethyl but-2-enolide & 12.17 & - & - & - & - & - & - & - & - & - & - & - & - & - & - & - & - & - & - & 0.05 & - \\
\hline Butyrolactone & 12.40 & - & - & - & - & - & - & - & - & - & - & - & - & - & - & - & - & - & 0.09 & - & - \\
\hline$\gamma$-Vinyl- $\gamma$-valerolactone & 12.90 & - & - & 0.41 & - & - & - & - & - & - & - & - & - & - & - & - & - & - & - & - & 1.13 \\
\hline$\gamma$-Caprolactone & 13.30 & - & - & - & Trace & - & - & - & - & - & - & - & - & - & - & - & - & 0.56 & 0.34 & - & - \\
\hline$\delta$-Octalactone & 16.22 & - & - & - & - & - & - & - & - & - & - & - & - & - & - & - & - & 0.62 & 0.26 & - & - \\
\hline$\delta$-Nonalactone & 18.41 & - & - & - & - & - & - & - & - & - & - & - & - & - & - & - & - & 0.10 & 0.26 & - & - \\
\hline 7-Decen-5-olide & 19.00 & 2.96 & - & - & - & - & - & - & - & - & - & - & - & - & - & - & - & 9.87 & 9.44 & - & - \\
\hline Total $\%$ & & 2.96 & - & 0.41 & Trace & - & - & - & - & - & - & - & - & - & - & - & - & 11.15 & 10.39 & 0.05 & 1.13 \\
\hline \multicolumn{22}{|l|}{ Nitrogen-bearing compounds } \\
\hline Methyl nicotinate* & 14.15 & - & - & - & - & - & - & - & - & - & - & - & - & - & - & - & 8.89 & - & - & - & - \\
\hline Phenylacetonitrile* & 15.88 & 0.12 & Trace & 0.06 & - & 0.05 & - & 0.04 & - & - & - & - & - & 1.52 & - & - & 0.54 & 2.18 & 0.22 & 0.06 & 0.42 \\
\hline Methyl anthranilate* & 18.93 & - & - & - & 0.10 & - & - & - & - & - & 31.31 & - & - & - & - & - & - & - & - & - & - \\
\hline Indole* & 20.76 & - & 0.48 & - & 0.26 & 0.45 & 0.30 & 0.02 & - & - & 15.07 & - & 1.68 & - & - & - & 0.09 & - & 0.03 & Trace & - \\
\hline Total $\%$ & & 0.12 & 0.49 & 0.06 & 0.36 & 0.49 & 0.30 & 0.06 & - & - & 46.38 & - & 1.68 & 1.52 & - & - & 9.52 & 2.18 & 0.25 & 0.06 & 0.42 \\
\hline Unknowns & & 0.10 & - & 0.36 & 0.12 & 0.24 & - & - & - & - & - & - & - & - & - & - & - & - & - & 1.77 & 0.30 \\
\hline
\end{tabular}

a Abbreviations: Acleisanthes acutifolia (Aac), A. crassifolia (Acr), A. longiflora (Alo), A. obtusa (Aob), A. wrightii (Awr), Mirabilis alipes (Mal), M. bigelovii (Mbi), M. greenei (Mgr), M. jalapa (Mja), M. longiflora (Mlo), M. macfarlanei (Mmf), M. multiflora (Mmu), M. pudica (Mpu), M. triflora (Mtr), Selinocarpus angustifolius (San), S. chenopodioides (Sch), S. lanceolatus (Sla), S. parvifolius (Spa), S. purpusianus (Spu), S. undulatus (Sun).

${ }^{b}$ Compounds within classes are listed according to retention time (RT); for each class its percentage of the total is given.

c Sample size $(n)$ is the number of individuals from which fragrance was collected; unless otherwise noted, only one fragrance collection per individual was included.

d Only one plant was available, but multiple collections were made of this plant.

${ }^{\mathrm{e}}$ Multiple collections from two plants were made during both the day and night for this diurnal species.

${ }^{\mathrm{f}}$ Total amount is the mean total unit area (as integrated peak area; see Experimental) of all volatiles produced per $12 \mathrm{~h}$, divided by $10^{6}$ for ease of presentation.

g $\%$ Floral is the percentage of the total volatiles emitted by flowers.

h The total number of both floral and vegetative compounds.

${ }^{\mathrm{i}}$ The total number of compounds produced solely or mainly from flowers.

$\mathrm{j}$ The amount of floral volatiles (an average of the total unit area) was adjusted to give the amount released per mg of floral tissue.

$\mathrm{k}$ Asterisks indicate compounds for which we have retention times from standards and agreement with mass spectral libraries.

${ }^{1}$ Trace $=<0.01 \%$ of volatiles emitted.

${ }^{\mathrm{m}}$ Entries in bold identify solely or mainly floral compounds.

${ }^{\mathrm{n}}$ Mass fragments for unknowns are listed with the molecular ion first (if known or inferred), followed by the base peak and other fragments in order of decreasing abundance.

1,5-Cyclo-undecadiene, 8,8-dimethyl-9-methylene.

p 12-Oxabicyclo 9.1.0 dodeca-3,7-diene,1,5,5,8-tetramethyl-,1R-(1R@,3E,7E,11R@). 
each genus separately is not monophyletic; all Mirabilis species also form a monophyletic group. Further, the amount of intraspecific variation varied among taxa. In particular, species that produce many different volatiles have necessarily more sources of variation and, thus, higher levels of intraspecific variation relative to other species. For example, the fragrance profile of $A$. wrightii contains the most compounds (Table 2), and this species also has one of the highest levels of intraspecific variation $($ mean $\%$ intraspecific dissimilarity $=23.5$, S.D. $=3.5)$. By contrast, Mirabilis species released fewer volatiles than Acleisanthes and Selinocarpus species and had fairly low levels of intraspecific variation (mean\% intraspecific dissimilarity $=8.8$, S.D. $=4.2)$ compared to Acleisanthes and Selinocarpus species (mean \% intraspecific dissimilarity $=14.1$, S.D. $=7.3$ ).

Further exploration of intraspecific variation can be accomplished by comparing our fragrance results for Mirabilis jalapa to those previously published (Heath and Manukian, 1994). Despite methodological differences (we sampled the fragrance from one individual whereas they sampled from 12) and selective reporting of fragrance compounds by Heath and Manukian (1994), the results of our two studies are generally consistent. Our study confirms the presence of trans- $\beta$-ocimene as the major component of the fragrance of M. jalapa $(38 \%$ of total volatiles emitted; Table 2). In further agreement with the previous study, we found cis-3-hexenyl acetate and myrcene. Our study did not detect benzaldehyde and indole; this may not be surprising, as Heath and Manukian (1994) report that neither of these compounds was found in all of their $M$. jalapa fragrance samples. We did find other compounds not reported by Heath and Manukian (1994), including $\alpha$-farnesene $(37 \%)$ and benzyl acetate (5\%) (Table 2).

\subsection{Volatiles in taxa with diurnal pollination}

The two species in this study known to be pollinated by animals other than moths (Table 1), Mirabilis triflora and M. macfarlanei, had low total volatile production (Table 2). One of these species, M. triflora, is hummingbird-pollinated. Hummingbirds have been shown to ignore fragrance while nectar-foraging (Bené, 1945; van Riper, 1960), and hummingbird-pollinated flowers are generally described as scentless (Faegri and van der Pijl, 1979). Nevertheless, flowers of M. triflora released some fragrance (Table 2). It is possible that volatile compounds that are no longer necessary for pollinator attraction may be retained for herbivore deterrence (Mullin et al., 1991; Berenbaum and Seigler, 1992; Dobson, 1994). However, as its closest relatives also produce fragrance (Levin, 2000), the emission of floral volatiles in M. triflora may suggest more about its phylogenetic heritage than an absence of strong selection against fragrance production due to its mode of pollination (Levin, 2001).
Mirabilis macfarlanei has been shown to be mainly bee-pollinated (Barnes, 1996). In addition to low overall volatile production, its flowers also released few compounds, with the monoterpene limonene as the main compound ( $43 \%$; Table 2 ). This compound is known to be perceived by bees (reviewed by Dobson, 1994), and in combination with visual cues it may be sufficient for attraction.

\subsection{Volatiles in moth-pollinated taxa}

The majority of species in this study are moth-pollinated (Table 1) and have a clearly discernible and detectable fragrance, but there are many quantitative and qualitative differences among them. One notable finding is that Acleisanthes and Selinocarpus species generally emit higher total amounts of volatiles than do Mirabilis species (Table 2). Also, there are substantial differences in the number of fragrance compounds emitted (Table 2), with Acleisanthes and Selinocarpus species averaging twice as many compounds $($ mean $=62)$ as Mirabilis species (mean $=24$; conservatively, the average number of compounds $=30$ when include only moth-pollinated Mirabilis and also exclude M. greenei, which has a very weak fragrance, see below).

In terms of biosynthetic classes, fragrances of Mirabilis species also are less diverse, with no species producing either phenylpropanoids or lactones (Table 2). The presence of compounds from these two biosynthetic classes appears to be phylogenetically informative, being characteristic of closely related species (Levin, 2000) within both Acleisanthes (phenylpropanoids) and Selinocarpus (lactones). Interestingly, most of the lactones observed do not appear to have been reported previously from floral fragrances (Knudsen et al., 1993).

In addition to the identity of individual fragrance compounds, the source of the volatiles should also be considered when comparing fragrance profiles among species, as both vegetative and floral volatiles are known to attract floral visitors (Raguso, 2001). For example, crepuscular hawkmoths respond to fragrance from close range $(0-10 \mathrm{~m})$ by approaching its source and then probing at bright objects (Brantjes, 1978; Raguso and Willis, in press); they do not functionally distinguish between floral and vegetatively-derived odors. Acleisanthes acutifolia and $A$. wrightii have very fragrant vegetation, with most of the total volatile production emanating from vegetation rather than from flowers (ca. 82\%; Table 2). Specifically, sesquiterpenoids are released in large amounts from the vegetation of both species. A similar situation is found in Selinocarpus angustifolius and S. undulatus, where the vegetation is characterized by the emission of high amounts of mono- and sesquiterpenoids. In addition to terpenoids, lipoxygenase-derived "green leaf volatiles" (mainly compounds with a cis-3-hexenyl moiety) are often released from vegetation (Hatanaka et al., 1986; 
Croft et al., 1993), and these compounds are also emitted vegetatively in $A$. acutifolia, A. wrightii, S. angustifolius and $S$. undulatus. Phylogenetic history may explain some of these patterns in fragrance chemistry (Levin, 2001). Interestingly, $S$. angustifolius and $S$. undulatus are strongly supported as sister species, and A. wrightii and A. acutifolia are also very closely related (Levin, 2000).

Whether floral or vegetative volatiles dominate the fragrance profile depends on the species; among all twenty species the volatiles emitted from flowers varied from $5-100 \%$ of the total amount of fragrance emitted (Table 2). Across all taxa some compounds are mainly emitted by flowers (Table 2). These floral compounds include the lactones and phenylpropanoids, the isoleucine-derived tiglates (Hill et al., 1980), cis-jasmone, the majority of the benzenoid compounds, and most of the nitrogen-bearing compounds.

The amount of each floral compound produced per $\mu \mathrm{g}$ of floral mass varies substantially among species (Table 2). Although compounds such as trans- $\beta$-ocimene, nerolidol, cis-jasmone, and methyl benzoate are emitted by flowers of many species, the amount produced can differ greatly. This finding suggests that simply the presence of these compounds, rather than their amounts, may be sufficient for moth attraction.

In general, our results are consistent with other studies that have detected floral fragrance in moth-pollinated taxa; however, the one exception is the absence of fragrance in Mirabilis greenei. In addition to its low volatile production, of the five volatile compounds collected from this species, only the homoterpene 4,8-dimethylnona-1,3,7-triene was likely to be floral, with the others emitted from the vegetation (Table 2). This lack of fragrance is surprising if this species is indeed moth-pollinated. However, any interpretation of the fragrance of $M$. greenei should be considered preliminary, as the fragrance samples from this species were collected in the field when temperatures were unusually low (these conditions were unique to this species' fragrance collection); low temperatures have been reported to inhibit volatile production (Jakobsen and Olsen, 1994).

\subsection{Fragrance and pollination}

Several authors have suggested a link between the presence of particular fragrance compounds and moth pollination (Nilsson et al., 1985; Kaiser, 1993; Knudsen and Tollsten, 1993; Miyake et al., 1998). Kaiser (1993) described moth-pollinated flowers as having a "whitefloral" odor, containing acyclic terpene alcohols such as linalool and nerolidol and simple aromatic alcohols like benzyl alcohol, 2-phenylethanol, and their esters. Jasmonates, tiglates, lactones, and nitrogen-bearing compounds are also prevalent in a variety of moth-pollinated flowers (Kaiser, 1993). Knudsen and Tollsten (1993) obtained results consistent with Kaiser (1993) in their survey of hawkmoth and noctuid moth-pollinated flowers among 15 species in 12 genera and 9 plant families, but they additionally suggested that hawkmoth-pollinated flowers can be distinguished from noctuid mothpollinated flowers by the presence of geraniolic compounds and oxygenated sesquiterpenes.

The fragrances of all of the moth-pollinated (i.e., both hawkmoth and noctuid moth-pollinated) species included in this study (except $M$. greenei) are similar in containing mono- and sesquiterpenoids, aliphatic compounds, benzenoids, and nitrogen-bearing compounds. These fragrance profiles are consistent with the descriptions of moth-pollinated fragrances reported by Kaiser (1993), and to some extent support the suggestion of Knudsen and Tollsten (1993) that the specific presence of oxygenated sesquiterpenes is more characteristic of hawkmothpollinated than noctuid moth-pollinated species. Of the 12 taxa in our study that are likely to be hawkmoth-pollinated, eight had fragrances that contained at least one oxygenated sesquiterpene. However, the fragrances of two likely noctuid moth-pollinated species, Selinocarpus angustifolius and $S$. undulatus, also contained oxygenated sesquiterpenes (Tables 1 and 2). Further, we observed that these two Selinocarpus species emitted the lowest total amount of volatiles and had the least diversity of compounds compared to other Selinocarpus. Based on floral morphology, Selinocarpus chenopodioides would appear to be noctuid moth-pollinated, but the white-lined sphinx moth (Hyles lineata) has also been observed visiting its flowers, and may move pollen (Table 1; Levin, 2001). Interestingly, flowers of this species emit a fragrance similar to that of hawkmoth-pollinated Selinocarpus species in that they produce large amounts of a variety of volatiles, including oxygenated sesquiterpenes (Table 2). Thus, while pollination by smaller insects may be occurring, the flowers are also attractive to hawkmoths. By contrast, the flowers of Mirabilis alipes, which are likely noctuid moth-pollinated, emit a low diversity of volatiles and lack oxygenated sesquiterpenoids. However, flowers of $M$. alipes also attract Hyles lineata, although the hawkmoths collected while visiting this species did not carry any pollen (Table 1; Levin, 2001). In general, results of this study suggest that a diversity of compounds may attract moths, and oxygenated sesquiterpenes, while commonly found in fragrances of hawkmoth-pollinated taxa, are not restricted to the fragrances of these species.

\subsection{Conclusions}

The fragrance profiles we observed in Nyctaginaceae are generally consistent with the "white-floral" odor described by Kaiser (1993) and the fragrances of mothpollinated plants reported by Knudsen and Tollsten (1993) and Miyake et al. (1998). However, the floral volatiles reported here and in previous studies are not unique to fragrances of hawkmoth-pollinated flowers; 
for example, many of these volatiles have also been reported from beetle-pollinated flowers (Azuma et al., 1997). In addition to the chemical similarities of fragrance across moth-pollinated taxa, there are many differences that give flowers of each species their distinctive odor. These include species-specific compounds (e.g., vanillin in $A$. wrightii and methyl nicotinate in $S$. chenopodioides), compounds shared by closely related species (e.g., $\delta$-octalactone and $\delta$-nonalactone in $S$. lanceolatus and $S$. parvifolius), and species-specific blends of more ubiquitous compounds.

Fragrance plays diverse roles in hawkmoth pollination, attracting moths from a distance (Tinbergen, 1958; Haber, 1984), combining with visual cues to elicit floral approaches and feeding (Brantjes, 1978; Raguso and Willis, 2001), and facilitating associative learning and discrimination (Daly and Smith, 2000). Among diverse hawkmoth-pollinated flowers, the presence of common classes of compounds, such as oxygenated terpenoids and aromatic esters, combined with the species-specific nature of each blend documented in our study and its antecedents, provides a complex ecological signal with the potential to support all of these functions.

\section{Experimental}

\subsection{Study taxa}

Fragrance was collected from multiple individuals (sample sizes are given in Table 2) of each of the 20 Nyctaginaceae species (Table 1), the majority of which are found in the Chihuahuan Desert in southwestern North America. These taxa were chosen because of the prevalence of hawkmoth pollination and multiple evolutionary losses of this pollination system (Levin, 2001). Vouchers of all species included in this study are housed in the herbarium at the University of Arizona (ARIZ; see Table 2 in Levin, 2000).

\subsection{Volatile collection}

Fragrance was collected in the field using dynamic head-space collection methods (see Raguso and Pellmyr, 1998, and references therein). Flowers on a living plant were enclosed within a polyacetate bag (Reynolds ${ }^{\circledR}$ oven bags) where volatile compounds emitted from the plant accumulated and were trapped in adsorbent cartridges through the use of a battery operated diaphragm pump (KNF Neuberger, Inc.). Glass cartridges were packed with $100 \mathrm{mg}$ of the adsorbent Porapak ${ }^{\mathbb{R}} \mathrm{Q}(80-100$ mesh), and the pump pulled air over the flowers and into the adsorbent trap at a flow rate of ca. $250 \mathrm{ml} / \mathrm{min}$. Fragrance collections typically commenced at floral anthesis and continued for $12 \mathrm{~h}$. Most of the species included in this study have nocturnal floral anthesis, such that fragrance collection usually began at dusk and ended early the following morning. As all these species have flowers that last only one day, this collection period generally encompassed the entire period during which a flower was open. For a few species, we were unable to collect floral fragrance in the field, and instead did so in the greenhouse using the same protocol as outlined above. In general one fragrance collection was made per individual plant, and the number of flowers included for each fragrance collection was noted. After fragrance collection, flowers were removed from the plants and weighed to obtain an average fresh wt per flower for each species.

Simultaneous collections of ambient and vegetative volatiles were used to distinguish between truly floral compounds, compounds emitted from the vegetation, and ambient contaminants. The architecture of these plants made it impossible to avoid trapping vegetative volatiles while also collecting volatiles from a number of flowers. Ideally the mass of vegetation from which fragrance was collected would have been quantified; however, this would have required destructive sampling of plants (in many cases fragrance was collected from an entire plant), which was not feasible.

\subsection{Chemical and data analysis}

After the $12 \mathrm{~h}$ collection period, cartridges were wrapped in aluminum foil and kept chilled. They were then eluted with $3 \mathrm{ml}$ of hexane, and the eluate was stored frozen in glass vials. Before GC-MS analysis, samples were concentrated to $75 \mu \mathrm{l}$ with $\mathrm{N}_{2}$. One microliter (occasionally $3 \mu \mathrm{l}$ ) aliquots of each sample were injected into a Shimadzu GC-17A equipped with a Shimadzu QP5000 quadrupole electron impact MS as a detector. All analyses were done using splitless injections on a polar GC column [diameter $0.25 \mathrm{~mm}$, length $30 \mathrm{~m}$, film thickness $0.25 \mu \mathrm{m}$ (EC WAX); Alltech Associates, Inc.]. The carrier gas was helium with a flow rate of $1 \mathrm{ml} / \mathrm{min}$ and a split ratio of 12 . The injector temp. was $240{ }^{\circ} \mathrm{C}$ and detector temp. was $260^{\circ} \mathrm{C}$. The oven program began with injection at $60{ }^{\circ} \mathrm{C}$ and a constant temp. for $3 \mathrm{~min}$. The temp. then increased by $10^{\circ} \mathrm{C}$ per min until $260^{\circ} \mathrm{C}$, where it was held for $7 \mathrm{~min}$. The column pressure at injection was 60.6 kilo Pascals $(\mathrm{kPa})$; the pressure then increased to $400 \mathrm{kPa}$ for $48 \mathrm{~s}$ followed by a decrease to $60.6 \mathrm{kPa}$ for $2 \mathrm{~min}$ (this pressure pulse was included for peak sharpening at the suggestion of L. Evanicke, Shimadzu Scientific Instruments, Inc.) and then an increase of $3.9 \mathrm{kPa}$ per min until $132.9 \mathrm{kPa}$, where it was held for 7 min.

Compounds were tentatively identified using computerized mass spectral libraries [Wiley and NIST libraries ( $>120,000$ mass spectra)]. The identity of many compounds was also verified using retention times of known standards (Table 2). To ensure that even small quantities 
of compounds were detected, we searched for the presence of specific mass fragments using single ion chromatograms (SIC). For example, the nitrogen-bearing compounds indole and phenylacetonitrile often were present in very small amounts; by searching for the molecular ion $(117 \mathrm{Da})$ at the appropriate retention times, we were able to detect trace amounts of these compounds. Quantification of compound amounts was achieved by integrating individual GC peak areas of the total ion chromatograms (TIC) using Shimadzu's Class-5000 software (Shimadzu Co., 1993-1996). For each species these compound areas were averaged across individuals and divided by the total average amount of volatiles produced to yield the relative amount (mean percentage) of each compound present in the floral and vegetative fragrance per $12 \mathrm{~h}$ collection period (Table 2). For volatiles released mainly or solely from flowers, compound areas were standardized by adjusting for the number and mass of flowers sampled to yield the amount present per $\mu \mathrm{g}$ of floral mass (Table 2).

\subsection{Intraspecific vs. interspecific variation}

To compare intraspecific variation in fragrance profiles to interspecific variation, we used the relative amounts of both floral and vegetative volatiles for each individual (rarely were multiple samples from the same individual included, see Table 2) to calculate a dissimilarity matrix based on Euclidean distance (SPSS Inc., 1999). Variables were standardized to have a mean of zero and a standard deviation of one. We then used a Wilcoxon Signed Rank Test (SPSS Inc., 1999) to compare the pair-wise $\%$ dissimilarities between individuals within a species to those between individuals among species. A lower dissimilarity between conspecific individuals than between individuals among species would suggest that interspecific variation is greater than intraspecific variation in fragrance profiles.

\section{Acknowledgements}

We are grateful to T. Tully, A. Boyd, M. Manktelow, D. Hearn, and A. Chetochine, without whose assistance these fragrance collections could not have been made. We thank Big Bend National Park, the Jornada LTER, and J. Duft for access to plants, and A. Le Duc and M. Fishbein for providing seeds. For use of a GC-MS and injection of many of the samples we thankfully acknowledge E. Pichersky and J. D'Auria, University of Michigan. This manuscript was greatly improved by J.S. Miller, who read an early version of the manuscript, and by three anonymous reviewers. The main source of funding for this research was provided by the National Science Foundation DEB9806840 to L.A.M. and R.A.R. Further support was provided by small grants to R.A.L. from the American Society of Plant Taxonomists, Sigma Xi, the Department of Ecology and Evolutionary Biology at the University of Arizona, and the University of Arizona Research Training Group in the Analysis of Biological Diversification (NSF DIR-9113362, BIR9602246). This paper is part of a dissertation by R.A.L. submitted in partial fulfillment of the requirements for the degree of doctor of philosophy, University of Arizona.

\section{References}

Azuma, H., Toyota, M., Asakawa, Y., Yamaoka, R., Garcia-Franco, J.G., Dieringer, G., Thien, L.B., Kawano, S., 1997. Chemical divergence in floral scents of Magnolia and allied genera (Magnoliaceae). Plant Species Biol. 12, 69-83.

Barkman, T.J., Beaman, J.H., Gage, D.A., 1997. Floral fragrance variation in Cypripedium: Implications for evolutionary and ecological studies. Phytochemistry 44, 875-882.

Barnes, J. L., 1996. Reproductive ecology, population genetics, and clonal distribution of the narrow endemic: Mirabilis macfarlanei (Nyctaginaceae). Master's thesis, Utah State University, Logan, Utah.

Barthlott, W., Porembski, S., Kluge, M., Hopke, J., Schmidt, L., 1997. Selenicereus wittii (Cactaceae): an epiphyte adapted to Amazonian Igapó inundation forests. Plant Syst. Evol. 206, 175-185.

Bené, F., 1945. The role of learning in the feeding behavior of Blackchinned Hummingbirds. The Condor 47, 3-22.

Berenbaum, M., Seigler, D., 1992. Biochemicals: engineering problems for natural selection. In: Roitberg, B.D., Isman, M.B. (Eds.), Insect Chemical Ecology: An Evolutionary Approach. Chapman \& Hall, New York, pp. 89-121.

Bestmann, H.J., Winkler, L., von Helversen, O., 1997. Headspace analysis of volatile flower scent constituents of bat-pollinated plants. Phytochemistry 46, 1169-1172.

Borg-Karlson, A.-K., Valterová, I., Nilsson, L.A., 1994. Volatile compounds from flowers of six species in the family Apiaceae: bouquets for different pollinators? Phytochemistry 35, 111-119.

Brantjes, N.B.M., 1978. Sensory responses to flowers in night-flying moths. In: Richards, A.J. (Ed.), The Pollination of Flowers by Insects. Academic Press, London, pp. 13-19.

Croft, K.P.C., Jüttner, F., Slusarenko, A.J., 1993. Volatile products of the lipoxygenase pathway evolved from Phaseolus vulgaris (L.) leaves inoculated with Pseudomonas syringae pv phaseolicola. Plant Physiol. 101, 13-24.

Cruden, R.W., 1970. Hawkmoth pollination of Mirabilis (Nyctaginaceae). Bull. Torr. Bot. Club 97, 89-91.

Daly, K., Smith, B.H., 2000. Associative olfactory learning in the moth Manduca sexta. J. Exp. Biol. 203, 2025-2038.

Dobson, H.E.M., 1994. Floral volatiles in insect biology. In: Bernays, E.A. (Ed.), Insect-Plant Interactions, Vol. V. CRC Press, Boca Raton, pp. 47-81.

Dobson, H.E.M., Arroyo, J., Bergström, G., Groth, I., 1997. Interspecific variation in floral fragrances within the genus Narcissus (Amaryllidaceae). Biochem. Syst. Ecol. 25, 685-706.

Dudareva, N., Pichersky, E., 2000. Biochemical and molecular genetic aspects of floral scents. Plant Physiol. 122, 627-633

Faegri, K., van der Pijl, L., 1979. The Principles of Pollination Ecology, 3rd Edition. Pergamon, Oxford

Fowler, B.A., Turner, B.L., 1977. Taxonomy of Selinocarpus and Ammocodon (Nyctaginaceae). Phytologia 37, 177-208.

Grant, V., 1983. The systematic and geographical distribution of hawkmoth flowers in the temperate North American flora. Bot. Gaz. 144, 439-449. 
Grant, V., Grant, K.A., 1983. Hawkmoth pollination of Mirabilis longiflora (Nyctaginaceae). Proc. Nat. Acad. Sci. 80, 12981299.

Grison, L., Edwards, A.A., Hossaert-McKey, M., 1999. Interspecies variation in floral fragrances emitted by tropical Ficus species. Phytochemistry $52,1293-1299$.

Haber, W.A., 1984. Pollination by deceit in a mass-flowering tropical tree Plumeria rubra L. (Apocynaceae). Biotropica 16, 269-275.

Haber, W.A., Frankie, G.W., 1989. A tropical hawkmoth community: Costa Rican dry forest Sphingidae. Biotropica 21, 155-172.

Hatanaka, A., Kajiwara, T., Sekiya, J., 1986. Fatty acid hydroperoxidase lyase in plant tissues: volatile aldehyde formation from linoleic and linolenic acid. In: Parliament, T.H., Croteau, R. (Eds.), Biogeneration of Aromas. American Chemical Society, Washington, DC, pp. 167-175.

Heath, R.R., Manukian, A., 1994. An automated system for use in collecting volatile chemicals released from plants. J. Chem. Ecol. 20, 593-607.

Hill, R.K., Rhee, S.-W., Leete, E., McGaw, B.A., 1980. Stereospecificity of enzymatic dehydrogenation during tiglate biosynthesis. J. Am. Chem. Soc. 102, 7344-7348.

Hodges, S.A., 1995. The influence of nectar production on hawkmoth behavior, self-pollination, and seed production in Mirabilis multiflora (Nyctaginaceae). Am. J. Bot. 82, 197-204.

Jakobsen, H.B., Olsen, C.E., 1994. Influence of climatic factors on emission of flower volatiles in situ. Planta 192, 365-371.

Kaiser, R., 1993. The Scent of Orchids: Olfactory and chemical investigations. Elsevier, Amsterdam.

Kaiser, R., Tollsten, L., 1995. An introduction to the scent of cacti. Flav. Fragr. J. 10, 153-164.

Knudsen, J.T., Tollsten, L., 1993. Trends in floral scent chemistry in pollination syndromes: floral scent composition in moth-pollinated taxa. Bot. J. Linn. Soc. 113, 263-284.

Knudsen, J.T., Tollsten, L., 1995. Floral scent in bat-pollinated plants: a case of convergent evolution. Bot. J. Linn. Soc. 119, 45-57.

Knudsen, J.T., Tollsten, L., Bergström, L.G., 1993. Floral scents-a checklist of volatile compounds isolated by head-space techniques. Phytochemistry 33, 253-280.

Levin, R.A., 2000. Phylogenetic relationships within Nyctaginaceae tribe Nyctagineae: evidence from nuclear and chloroplast genomes. Systematic Botany 25, 738-750.

Levin, R.A., 2001. Relationships among fragrance, phylogeny and pollination in southwestern Nyctaginaceae. PhD. diss. University of Arizona, Tucson, AZ.

Martínez Del Río, C., Búrquez, A., 1986. Nectar production and temperature dependent pollination in Mirabilis jalapa L. Biotropica $18,28-31$.
Miyake, T., Yamaoka, R., Yahara, T., 1998. Floral scents of hawkmoth-pollinated flowers in Japan. J. Plant Res. 111, 199-205.

Mullin, C.A., Alfatafta, A.A., Harman, J.L., Serino, A.A., Everett, S.L., 1991. Corn rootworm feeding on sunflower and other Compositae: influence of floral terpenoid and phenolic factors. In: Hedin, P.A. (Ed.), Naturally Occurring Pest Bioregulators. American Chemical Society, Washington, DC, pp. 278-292.

Nilsson, L.A., Jonsson, L., Rason, L., Randrianjohany, E., 1985. Monophily and pollination mechanisms in Angraecum arachnites Schltr. (Orchidaceae) in a guild of long-tongued hawk-moths (Sphingidae) in Madagascar. Biol. J. Linn. Soc. 26, 1-19.

Pilz, G.E., 1978. Systematics of Mirabilis subgenus Quamoclidion (Nyctaginaceae). Madroño 25, 113-132.

Radwanski, E.R., Last, R.L., 1995. Tryptophan biosynthesis and metabolism: biochemical and molecular genetics. The Plant Cell 7, 921-934.

Raguso, R.A., 2001. Floral scent, olfaction and scent-driven foraging behavior. In: Chittka, L., Thomson, J. (Eds.), Cognitive Ecology of Pollination: Animal Behavior and Floral Evolution. Cambridge University Press, Cambridge, pp. 83-105.

Raguso, R.A., Pellmyr, O., 1998. Dynamic headspace analysis of floral volatiles: a comparison of methods. Oikos 81, 238-254.

Raguso, R.A., Pichersky, E., 1995. Floral volatiles from Clarkia brewerii and C. concinna (Onagraceae): recent evolution of floral scent and moth pollination. Plant Syst. Evol. 194, 55-67.

Raguso, R.A., Willis, M.W., 2001. The importance of olfactory and visual cues in nectar foraging by nocturnal hawkmoths. In: Boggs, C.L., Watt, W.B., Ehrlich, P.R. (Eds.), Proceedings of the Third Symposium on Butterfly Ecology and Evolution. University of Chicago Press, Chicago, in press.

Shimadzu Co., 1993-1996. Class-5000, Version 2.0.0. Shimadzu Chemical Laboratory Analysis System \& Software.

Spellenberg, R., Delson, R. K., 1977. Aspects of reproduction in Chihuahuan Desert Nyctaginaceae. In: Wauer, R. H., Riskind, D. H. (Eds.), Transactions of the Symposium on the Biological Resources of the Chihuahuan Desert Region United States and Mexico. US Department of the Interior, pp. 273-287.

SPSS Inc., 1999. SPSS for Windows, Version 10.0.5. SPSS Inc., Chicago. Tinbergen, N., 1958. Curious Naturalists. Doubleday \& Co., Garden City, NY.

van Riper, W., 1960. Does a hummingbird find its way to nectar through its sense of smell? Sci. Am. 202, 157-166.

Wang, J., Pichersky, E., 1999. Identification of specific residues involved in substrate discrimination in two plant $O$-methyltransferases. Arch. Biochem. Biophys. 368, 172-180.

Wink, M., 1997. Special nitrogen metabolism. In: Dey, P.M., Harborne, J.B. (Eds.), Plant Biochemistry. Academic Press, San Diego, pp. 439486. 